\title{
An event-related potential study on the observation of erroneous everyday actions
}

\author{
ELLEN R. A. DE BRUIJN \\ Max Planck Institute for Human Cognitive and Brain Sciences, Leipzig, Germany \\ and Radboud University Nijmegen, Nijmegen, The Netherlands \\ RICARDA I. SCHUBOTZ \\ Max Planck Institute for Human Cognitive and Brain Sciences, Leipzig, Germany \\ and Otto von Guericke University of Magdeburg, Magdeburg, Germany \\ AND \\ MARKUS UllsPerger \\ Max Planck Institute for Human Cognitive and Brain Sciences, Leipzig, Germany \\ and Max Planck Institute for Neurological Research, Cologne, Germany
}

\begin{abstract}
Recent experiments have demonstrated that error-related negativity (ERN) is not only elicited when people commit errors, but also when they observe others committing errors. The present study investigates whether this observed ERN is also present when participants observe execution errors in an everyday context. Participants observed short sequences of pictures showing steps of everyday actions ending either erroneously or correctly. Participants were instructed to indicate by a delayed response whether the observed action was correctly executed or not. The results showed a large P300 for execution errors compared with the observation of correct sequences, but no ERN activity was found. The present experiment indicates that the detection of execution errors in observation does not rely on the error processing mechanism responsible for generating the ERN. The increased P300 amplitudes suggest a more general monitoring process that signals that the occurrence of unexpected events is involved in the detection of execution errors.
\end{abstract}

It is crucial for humans to adapt their behavior quickly to a continuously changing environment. To accomplish this, it is not only important to monitor one's own actions, but also to act upon external feedback related to them. Additionally, when working with another person, it is relevant to monitor that person's intentions and actions as well. These different aspects of action monitoring have been investigated over the past decade by means of event-related potentials (ERPs). A specific component in the response locked ERP, the error-related negativity (ERN; Falkenstein, Hohnsbein, Hoormann, \& Blanke, 1990; Gehring, Goss, Coles, Meyer, \& Donchin, 1993) has been shown to be associated with errors in speeded choice-reaction tasks. In their reinforcement-learning theory of the ERN, Holroyd and Coles (2002) proposed that the ERN is generated when a prediction error signal that is carried by dopaminergic pathways arrives in the posterior medial frontal cortex (pMFC).

More recently, a so-called observed ERN has been found when a participant observes another person making an error (Bates, Patel, \& Liddle, 2005; Miltner, Brauer, Hecht, Trippe, \& Coles, 2004; van Schie, Mars, Coles, \& Bekkering, 2004). van Schie and colleagues localized the source for both the ERN following own errors and the
ERN after observed errors in nearby areas in the pMFC. On the basis of this finding, it was suggested that similar neural processes are involved in the detection of one's own errors, as well as of other people's.

A possible limitation of the employed tasks in previous experiments is that they are rather symbolic in nature. Speeded choice-reaction tasks lead to the commission of errors that are the direct result of incorrect choices. As such, the majority of ERN research so far has focused on discrete choice-errors (but see de Bruijn, Hulstijn, Meulenbroek, \& van Galen, 2003; Krigolson \& Holroyd, 2006; Luu, Flaisch, \& Tucker, 2000). However, the actions that people perform in everyday life are very different and tend to be more continuous. Errors in these actions often result not from incorrect choices but from an inaccurate execution of the selected action. As such, execution errors in everyday actions are generally unexpected events. One of the main assumptions of the reinforcement-learning theory is that the least expected error elicits the largest ERN (Holroyd \& Coles, 2002). Therefore, in the present experiment, we investigate whether an observed ERN is also elicited when participants watch other people commit errors in the course of performing everyday actions.

E. R.A. de Bruijn, ellendb@nici.ru.nl 


\section{METHOD}

\section{Participants}

Eighteen healthy volunteers ( 8 male, 10 female; mean age, 22.7 years) participated in the experiment after their written informed consent had been obtained. They were paid for participation.

\section{Task}

Participants observed 80 short sequences of six pictures showing steps of actions such as watering plants or cutting paper with scissors (see Figure 1 for two examples and trial duration). In 50\% of the trials, the presented action ended in an execution error. Each action sequence had both a correct and an erroneous ending. These endings were distributed over participants, such that each participant observed each action sequence only once in either the correct or the erroneous version. Participants were instructed to indicate by a delayed response whether the observed action was executed correctly or not. The next trial would start $100 \mathrm{msec}$ after the given response. Note that the stimulus material was tested beforehand in a pilot study in which participants had to indicate after each picture whether the action sequence would end in an error or in a correct response. Importantly, this pilot study demonstrated that predictions were at chance level until the fifth picture; therefore, it was only the sixth picture which disambiguated the course of the action.

All participants additionally performed a modified flanker task (Eriksen \& Eriksen, 1974), consisting of 960 trials. Participants were presented for about $500 \mathrm{msec}$ with a fixation mark at the center of a screen, after which four flanker arrows $-0.46^{\circ}$ tall and $1.08^{\circ}$ wide, appearing $0.52^{\circ}$ and $1.04^{\circ}$ above and below the screen center-were shown for $110 \mathrm{msec}$. The target arrow was presented for $30 \mathrm{msec}$ in the center of the flanker arrows; its onset was delayed by $80 \mathrm{msec}$ from the flanker's onset. Participants had to press the left or right re- sponse button, according to the direction of a centrally presented target arrow. The distracting flanker arrows presented above and below the target were compatible (50\%) or incompatible $(50 \%)$ with the required response. At 1,400 msec after target onset, each response was followed by a symbolic feedback $(600 \mathrm{msec})$ informing participants whether or not their answers were fast enough. After the feedback, a fixation cross was presented for $500 \mathrm{msec}$, such that the intertrial interval amounted to $2,580 \mathrm{msec}$. We employed an adaptive algorithm, which dynamically adjusted the response-time (RT) pressure on the basis of the participant's performance (Fiehler, Ullsperger, \& von Cramon, 2005). The algorithm aimed at an optimization of error rate (goal: $20 \%$ incompatible errors) and a minimization of late response rates. This procedure helped to reduce dropouts for a low number of error trials. Both the action-observation task and the flanker task were performed within one session in a counterbalanced fashion.

\section{Electrophysiological Recording and Data Analysis}

The EEG signal was recorded from $64 \mathrm{Ag} / \mathrm{AgCl}$ electrodes mounted in an elastic electrode cap (Easycap, Herrsching, Germany). All signals were later rereferenced offline to the average of both mastoids. The vertical electrooculogram (EOG) was recorded bipolarly from electrodes placed above and below the right eye. The horizontal EOG was also recorded bipolarly from electrodes lateral to both eyes. All electrode impedances were kept below $5 \mathrm{k} \Omega$. The EEG and EOG signals were amplified using BrainAmps MR plus amplifiers (BrainProducts, Munich) and were filtered offline lowpass at $12 \mathrm{~Hz}$. All signals were digitized with a sampling rate of $250 \mathrm{~Hz}$. EOG artifact correction was carried out using the procedure of Gratton, Coles, and Donchin (1983).

For the action-observation task, the ERPs were time locked to the onset of the last picture and averaged separately for correct sequences and execution errors relative to a $200-\mathrm{msec}$ prestimulus

\section{Correct sequence}

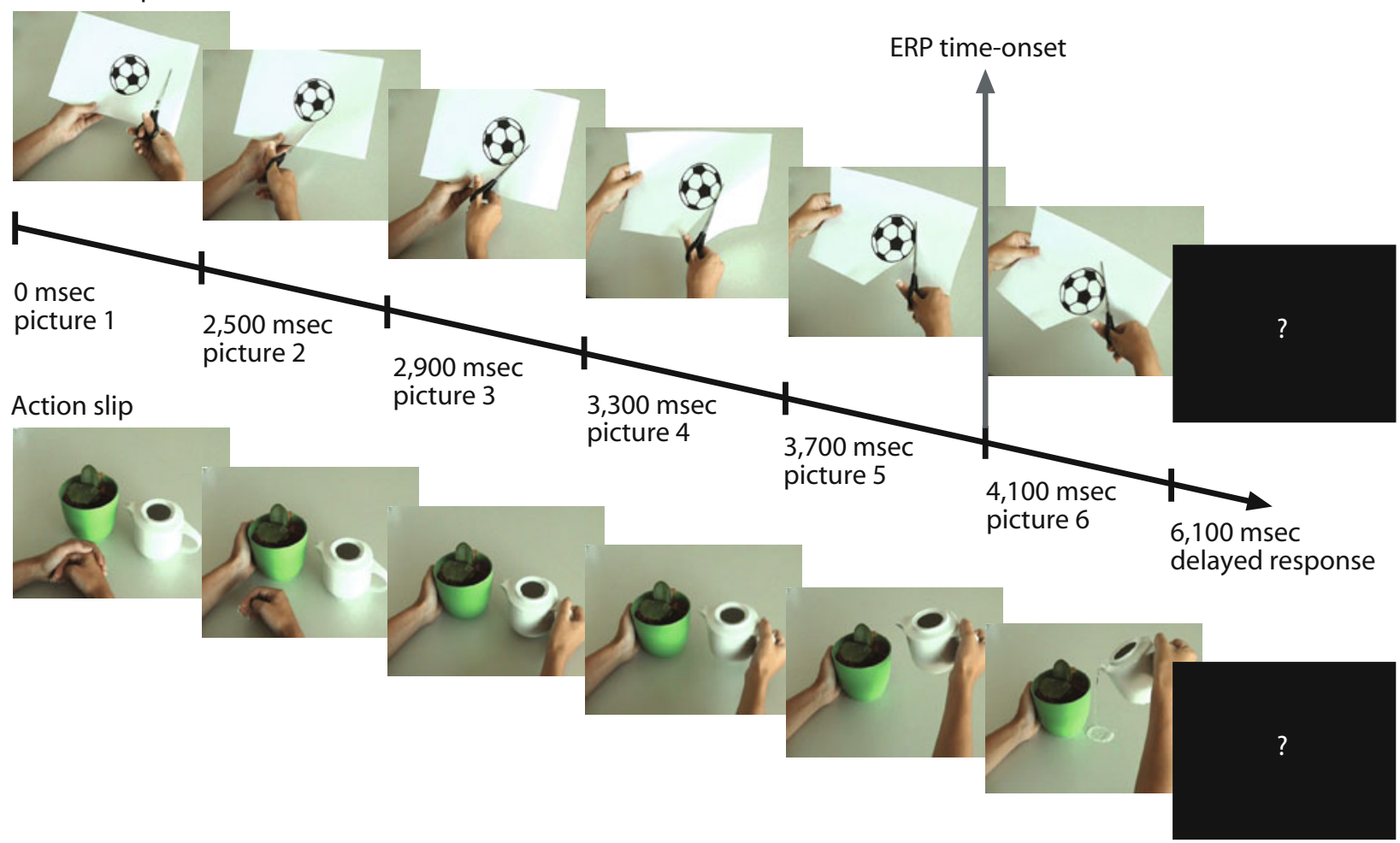

Figure 1. Examples of a correct sequence (upper panel) and an execution error (lower panel). ERPs were time locked to the onset of Picture 6, always containing the crucial information about the correctness of the observed action. Two seconds after presentation of this last picture, a question mark appeared signaling participants to respond. 
baseline. Observed-ERN amplitude was defined as the most negative peak in the 200 - to 350 -msec time window following stimulus onset at electrode $\mathrm{FCz}$, where maximal ERN amplitudes were expected (Bates et al., 2005). P300 amplitude was defined as the most positive peak in the 200- to 800 -msec time window following stimulus onset at electrode $\mathrm{Pz}$.

For the flanker task, the ERPs were time locked to response onset and averaged separately for correct and incorrect responses relative to a $200 \mathrm{msec}$ preresponse baseline. ERN amplitude was defined as the difference between the most negative peak in the 0 - to 200 -msec time window after response onset and the most positive peak in the time window starting $80 \mathrm{msec}$ before and ending $80 \mathrm{msec}$ after response onset at electrode $\mathrm{FCz}$, where maximal ERN amplitudes were expected (Falkenstein, Hoormann, Christ, \& Hohnsbein, 2000). Note that only responses to incompatible stimuli were analyzed.

Finally, previous studies have suggested that the ERN may arise from error induced increase of ongoing midline frontal theta (4-7 Hz) EEG activity (Luu \& Tucker, 2001; Luu, Tucker, \& Makeig, 2004; Yordanova, Falkenstein, Hohnsbein, \& Kolev, 2004). Although a total theta power increase time locked to the erroneous response has been found in a single trial analysis (Debener et al., 2005), it is still debated whether the ERN is at least partly a result of a theta phase resetting on erroneous responses (Yeung, Bogacz, Holroyd, Nieuwenhuis, \& Cohen, 2007). Nevertheless, ERN activity can be disentangled from possible P300 component overlap by filtering the single trial ERP data with a bandpass $4-7 \mathrm{~Hz}$ digital filter (see, e.g., Gehring \& Willoughby, 2004; Luu et al., 2004). To allow for theta-band-specific analyses, we filtered the data from both tasks accordingly. After averaging, the ERN peak was defined at FCz as the difference between the most negative peak in the 200- to 350-msec time window after stimulus onset and its preceding positive peak for the action-observation task, and as the difference between the most negative peak in the 0 - to $200-\mathrm{msec}$ time window after response onset and its preceding positive peak for the flanker task (Falkenstein et al., 2000).

For both tasks, individual averages for RTs and amplitudes were entered in a repeated measures general linear model (GLM) with correctness (2 levels: correct sequence/correct response vs. execution error/incorrect response) as a within-subjects factor.

\section{RESULTS}

\section{Action-Observation Task}

All trials with reaction times larger than $725 \mathrm{msec}$ were considered late responses and were removed from both the behavioral and the ERP analyses $(12.0 \%$, standard deviation $[S D]=10.0 \%$ ). This was done to rule out confounds with high decision uncertainty and to focus on those trials in which participants could classify the action outcome unequivocally. Also, action sequences that were erroneously categorized by participants were removed from both datasets $(7.6 \%, S D=4.9 \%)$. For the remaining trials (mean trial numbers: for correct actions, 33; for incorrect actions, 31), the RT analyses revealed faster RTs for the categorization of execution errors $(394 \mathrm{msec}$, $S D=101 \mathrm{msec}$ ) than for correct sequences $[408 \mathrm{msec}$, $S D=92 \mathrm{msec} ; F(1,17)=5.46, p=.032]$. Figure $2 \mathrm{de}-$ picts the grand-average ERP waveforms for the actionobservation task. With regard to ERN amplitude, no main effect for correctness was found, indicating that correct sequences $(-1.60 \mu \mathrm{V})$ and execution errors $(-1.43 \mu \mathrm{V})$ did not differ $[F(1,17)=0.15, p=.708]$. With regard to P300 amplitude, a main effect for correctness was present $[F(1,17)=95.29, p<.001]$, indicating that the $\mathrm{P} 300$ was significantly increased for execution errors $(8.60 \mu \mathrm{V})$ compared with correct sequences $(3.47 \mu \mathrm{V})$. Figure $3 \mathrm{~A}$ shows the topographical distribution of the P300 following the observation of execution errors.

\section{Flanker Task}

As in similar studies, incorrect responses were faster (299 $\mathrm{msec}, S D=26 \mathrm{msec})$ than correct responses [402 msec, $S D=20 \mathrm{msec} ; F(1,17)=262.06, p<.001$ ]. Participants responded incorrectly to $20.6 \%$ ( $S D=$ $5.8 \%$ ) of all incompatible trials. As can be seen in Figure 4, the ERN is present following incorrect responses $(-18.35 \mu \mathrm{V})$ but not, or to a lesser extent, after correct responses $[-8.08 \mu \mathrm{V} ; F(1,17)=52.04, p<.001]$. Figure $3 \mathrm{~B}$ shows the topographical distribution of the ERN following incorrect responses.

\section{Theta-Band Analyses}

Figure 5 depicts the theta-band-filtered grand-average waveforms for both tasks. Visual inspection of the waveforms suggests that midline theta oscillations are visible in the action-observation task as well as in the flanker task. However, the theta-band-filtered ERN is very pronounced in the flanker task, but not - or to a lesser extent - in the action-observation task. Subsequent analyses confirmed this observation. ERN amplitude did not differ significantly between correct sequences $(-2.02 \mu \mathrm{V})$ and execution errors $(-2.39 \mu \mathrm{V})$ in the action-observation task $[F(1,17)=0.88, p=.361]$. In contrast, in the flanker task theta-band-filtered ERN amplitudes were larger for incorrect responses $(-13.70 \mu \mathrm{V})$ than for correct responses $[-4.75 \mu \mathrm{V} ; F(1,17)=91.63, p<.001]$.

\section{DISCUSSION}

The present data show that no ERN-like activity was found for the observation of erroneous everyday actions, although the same participants did show a distinct frontocentrally oriented response ERN in the flanker task. Instead, a large P300 with a more posterior distribution is present following the observation of action slips compared with correctly executed actions. Moreover, the analyses on the theta-band-filtered ERP data demonstrate that the large P300 is not simply overshadowing any underlying ERN activity. This is an indication that the monitoring system responsible for generating the ERN is not significantly activated by the observation of execution errors.

The absence of an ERN is in line with the idea that the component is only elicited when there is a need for adjustments after the detection that the present outcome is worse than expected (see, e.g., Holroyd \& Coles, 2002; M. Ullsperger, Volz, \& von Cramon, 2004). Participants had to categorize the observed actions on the basis of correctness, but the consequences of the observed actions were not relevant for their own performance. Although previous studies that did demonstrate an observed ERN did not include an explicit error consequence for the observer either-e.g., a loss of reward - it may be argued that participants were more involved in those observation conditions, since they additionally performed the same task themselves. 

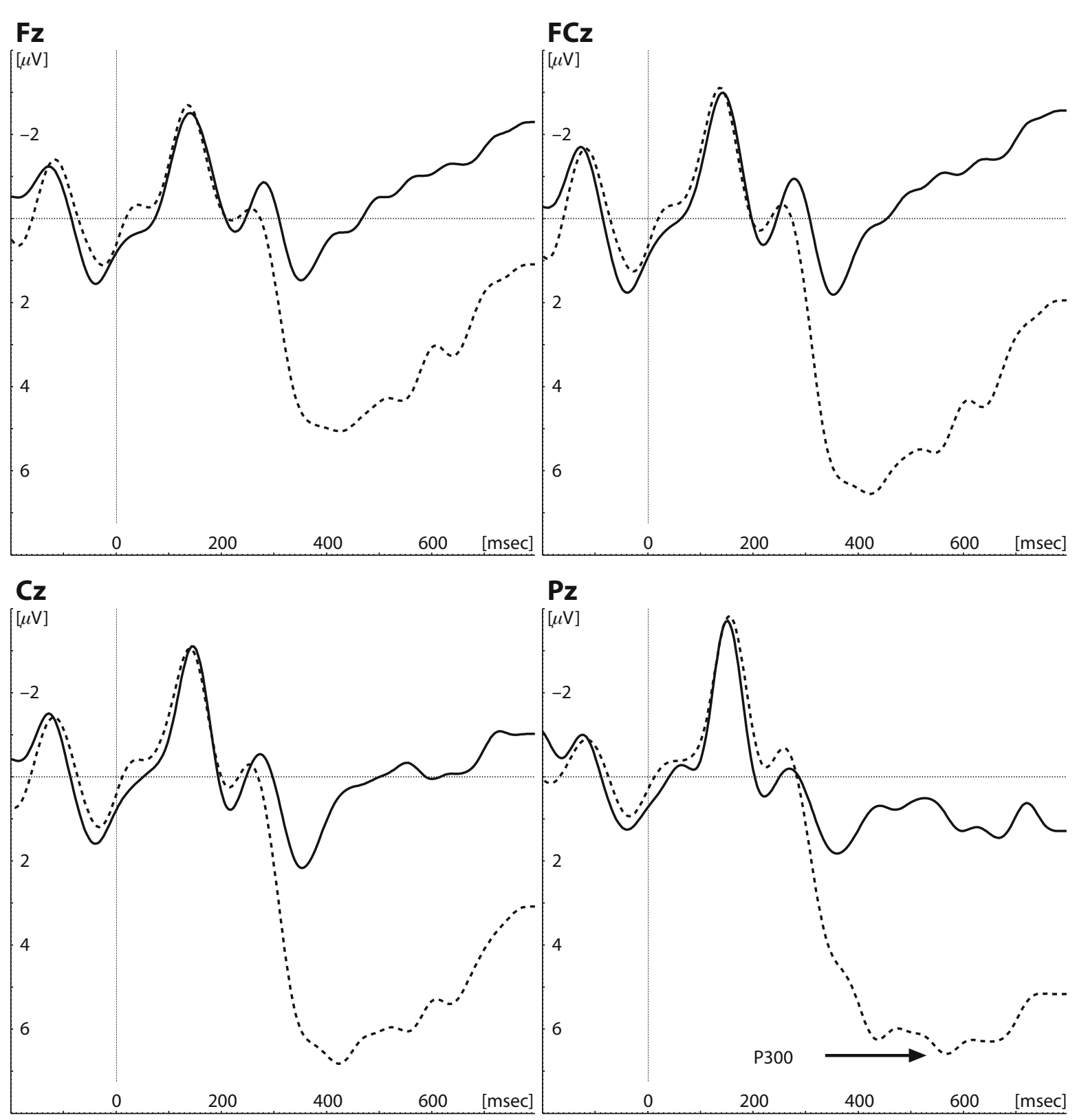

Figure 2. Grand-average ERP waveforms for the action-observation task time locked to the onset of the last picture ( $t=$ 0 msec) for correct sequences (solid lines) and execution errors (dashed lines).

A major difference from previous studies is that the present task was not a discrete speeded choice-reaction task. The continuous nature of the actions in the present experiment may have induced a stronger expectancy buildup. Although the overall probability for observing either a correct or an erroneous action was 50/50, participants may have had a stronger subjective expectancy for one of the two. Although it is difficult to draw conclusions from the present data, it is plausible that participants had a within-trials bias for expecting a correct action. The present design may have induced a stronger bias to correct actions, due to the longer presentation time of the first picture of the action sequence; as a result of this longer presentation time, participants would try to interpret and predict the upcoming action. Compared with correct action sequences, possible error scenarios were numerous and often highly unpredictable. As a result, participants were more likely to predict the correct action that was about to be performed from the start of the sequence. In a manner similar to real-life situations, expectancies were thus stronger for correct actions than for execution errors. Conversely, in previously used discrete choice-reaction tasks, participants were always aware of the highly limited set of possible response options (e.g., left vs. right response, or go vs. no-go response). As a result, erroneous responses in choice-reaction tasks are actually an- 

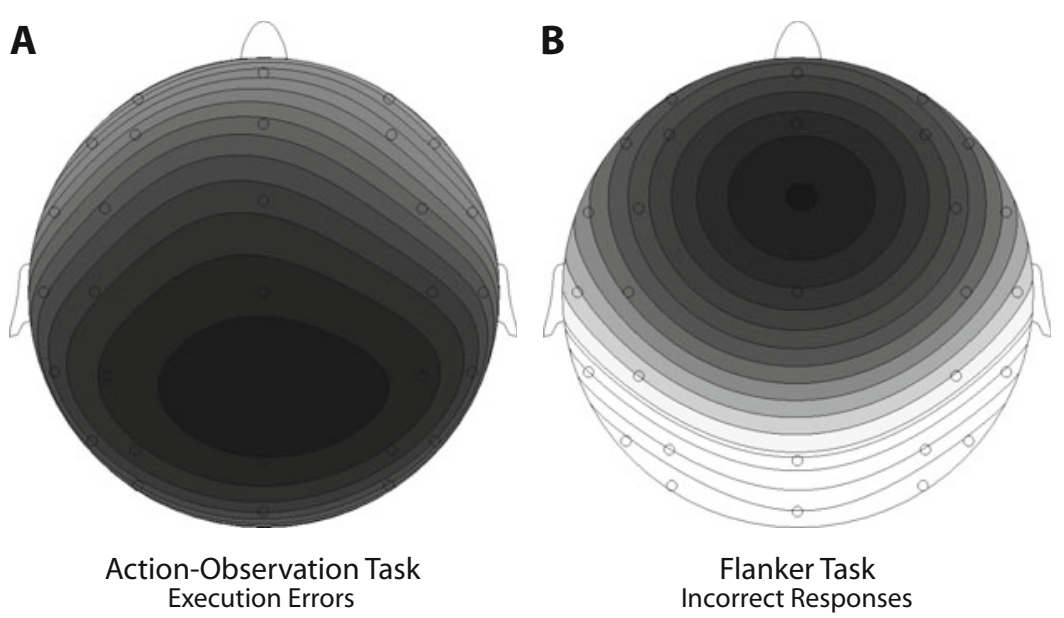

Figure 3. Topographical distribution (top view) of the P300 and ERN. (A) Parietal P300 in the action-observation task at 568 msec (scale: 1 to $6 \mu \mathrm{V}$, light to dark). (B) ERN in the flanker task at $52 \mathrm{msec}$ (scale: -13 to $4 \mu \mathrm{V}$, dark to light).

ticipated events, whereas execution errors in everyday tasks are mostly surprising events. The P300 has been suggested to reflect updating of contextual information in working memory (Donchin \& Coles, 1988; Mecklinger \& Ullsperger, 1995). Its amplitude seems to be related to the mental distance to an adaptation level acquired on the basis of the history of preceding events (P. Ullsperger \& Mecklinger, 1996). The large P300 amplitudes therefore suggest that a more general monitoring process is activated, one not specifically associated with error processing but with the detection of salient and unexpected events relevant for action selection in the task at hand. As the subjective expectancy bias remains difficult to determine, future experiments may focus on the investigation of different probability distributions for correct and incorrect actions in order to unravel the mechanisms underlying the currently found P300 findings in error observation.

The finding that the error monitoring system responsible for generating the ERN is currently not involved may also suggest that the presumed generator of the ERN, the pMFC (for a review, see Ridderinkhof, Ullsperger, Crone, \& Nieuwenhuis, 2004), is not implicated in the detection of observed execution errors. This is also suggested by the different scalp topographies for the two tasks showing that the P300 elicited by execution errors has a more posterior distribution than the frontocentrally located ERN in the flanker task. Note that in the present experiment, sequences of actions were presented using pictures to ensure a discrete time point to which the EEG signal could be locked for averaging. It may be argued that this method might bias the results and that observation of continuous movie clips would elicit an ERN. However, this is highly unlikely, since a previous fMRI study that did use continuous movie clips did not show an increase in pMFC activation for the observation of execution errors compared with correct actions, either (Manthey, Schubotz, \& von Cramon, 2003). Moreover, earlier ERP studies have demonstrated that observing real actions is not a prerequisite for activating the pMFC in response to errors as reflected in the presence of an ERN. Medial frontal negativities sharing features with the feedback ERN, for example, are known to be elicited by visual feedback stimuli in the absence of any action (see, e.g., Donkers, Nieuwenhuis, \& van Boxtel, 2005). Therefore, it is reasonable to assume that the currently applied methodology of presenting sequences of pictures cannot be why the present results do not demonstrate an ERN.

The lack of involvement of the pMFC is also supported by recent studies suggesting a dissociation between the systems in the brain responsible for monitoring errors in movement execution ("low-level" errors) and errors in choice ("high-level" errors) (de Bruijn et al., 2003; Krigolson \& Holroyd, 2006). Although it is generally agreed that the pMFC is involved in monitoring violations of highlevel goals (e.g., choice errors in RT tasks, or negative feedback in reinforcement-learning paradigms), the posterior parietal cortex is thought to enable the online control of movement (Desmurget et al., 1999; Desmurget et al., 2001), and could thus be specifically concerned with lowlevel error monitoring (Krigolson \& Holroyd, 2006). As such, the present data might, on the other hand, be in line with involvement of a more posterior error-monitoring system for the observation of execution errors.

Obviously, the present action-observation task and previous choice-reaction tasks differ with respect to performance measures. The number of execution errors in the observation task (mean $=31$ ) for example, is relatively small and lower than the absolute number of errors in the present flanker task (mean $=98)$. However, we do not believe that the low error rate in the action-observation task affected the present results, since previous studies have repeatedly demonstrated that comparable or even lower error rates also elicit an ERN (see, e.g., Debener et al., 2005; de Bruijn, Hulstijn, Verkes, Ruigt, \& Sabbe, 2004).

Conclusions about dopaminergic involvement in the observation of execution errors cannot be drawn from the present results. The present study shows that an ERN is 

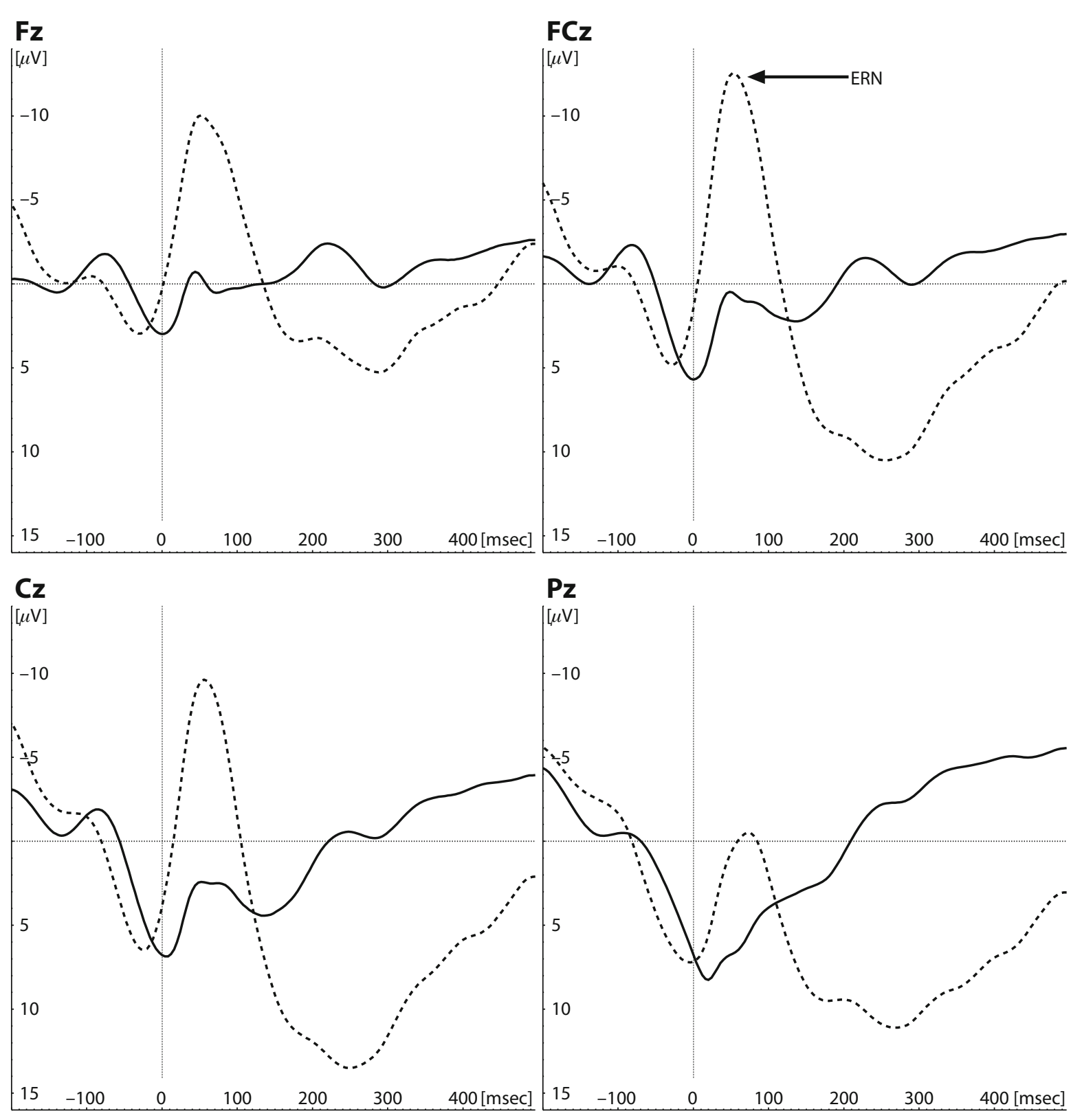

Figure 4. Grand-average ERP waveforms for the flanker task time locked to the onset of the response $(t=0 \mathrm{msec})$ for correct responses (solid lines) and incorrect responses (dashed lines).

not generated during the observation of execution errors. Although neurobiological considerations and computational models suggest a direct link between phasic dopamine signals and the generation of the ERN in humans (Holroyd \& Coles, 2002), direct evidence supporting this assumption is still lacking. Therefore, the question of whether observed execution errors may elicit a dopamine response similar to that which appears to be elicited by errors and negative feedback remains unanswered.

To conclude, the present experiment demonstrates that the detection of execution errors in observation does not rely on the error processing mechanism responsible for generating the ERN. The increased P300 amplitudes sug- gest that a more general monitoring process that signals the occurrence of unexpected task-relevant events is involved in the detection of observed execution errors. Our results are also in agreement with the recently proposed distinction between a posterior low-level error-monitoring system and a frontal high-level error-monitoring system. Previous studies strongly suggested that the pMFC was concerned with detecting high-level errors, regardless of whether these were executed or observed. Analogously, one may argue that - since execution errors are mostly low-level errors - the detection of observed execution errors is also mediated by a posterior error-monitoring system. 


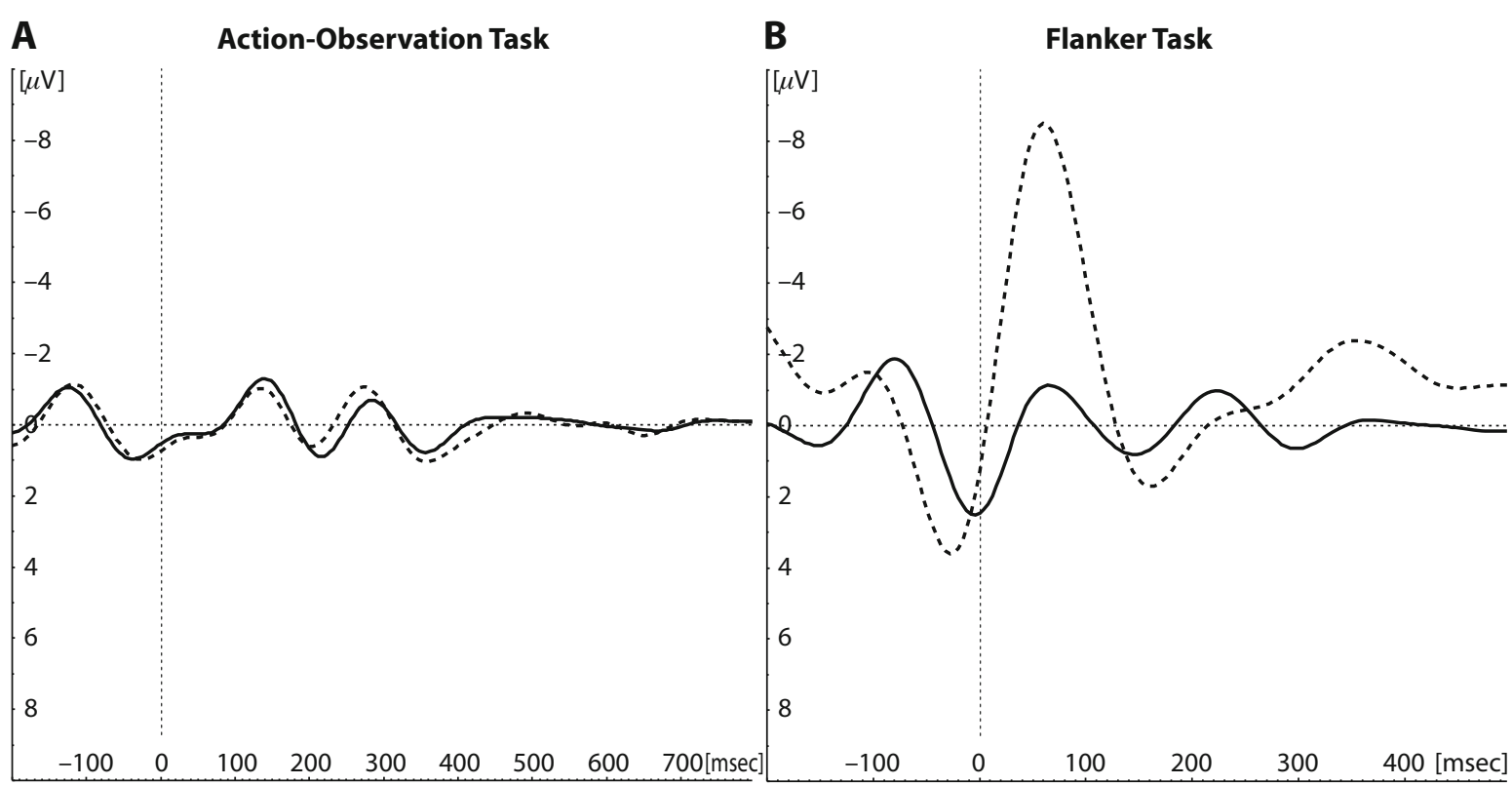

Figure 5. Grand-average ERP waveforms for the theta-band-filtered data at electrode FCz. The ERPs are time locked to $t=$ 0 msec, which corresponds to stimulus onset for the action-observation task (A) and to response onset for the flanker task (B). Correct responses (solid lines) and incorrect responses (dashed lines) are depicted.

\section{AUTHOR NOTE}

E.R.A.d.B. was supported by a grant from the Niels Stensen Foundation and by the EU-Project "Joint Action Science and Technology" (IST-FP6-003747). Correspondence concerning this article should be addressed to E. R. A. de Bruijn, Nijmegen Institute for Cognition and Information, Radboud University Nijmegen, 6500 Nijmegen, The Netherlands (e-mail: ellendb@nici.ru.nl).

\section{REFERENCES}

Bates, A. T., Patel, T. P., \& Liddle, P. F. (2005). External behavior monitoring mirrors internal behavior monitoring. Journal of Psychophysiology, 19, 281-288.

Debener, S., Ullsperger, M., Siegel, M., Fiehler, K., von Cramon, D. Y., \& ENGEL, A. K. (2005). Trial-by-trial coupling of concurrent electroencephalogram and functional magnetic resonance imaging identifies the dynamics of performance monitoring. Journal of Neuroscience, 25, 11730-11737.

de Bruijn, E. R. A., Hulstijn, W., Meulenbroek, R. G. J., \& van Galen, G. P. (2003). Action monitoring in motor control: ERPs following selection and execution errors in a force production task. Psychophysiology, 40, 786-795.

de Bruijn, E. R. A., Hulstijn, W., Verkes, R. J., Ruigt, G. S. F., \& SABBE, B. G. C. (2004). Drug-induced stimulation and suppression of action monitoring in healthy volunteers. Psychopharmacology, 177, 151-160.

Desmurget, M., Epstein, C. M., Turner, R. S., Prablanc, C., AlEXANDER, G. E., \& Grafton, S. T. (1999). Role of posterior parietal cortex in updating reaching movements to a visual target. Nature Neuroscience, 2, 563-567.

Desmurget, M., Gréa, H., Grethe, J. S., Prablanc, C., Alexander, G. E., \& Grafton, S. T. (2001). Functional anatomy of nonvisual feedback loops during reaching: A positron emission tomography study. Journal of Neuroscience, 21, 2919-2928.

Donchin, E., \& Coles, M. G. H. (1988). Is the P300 component a manifestation of context updating? Behavioral \& Brain Sciences, 11, 357-427.

Donkers, F. C. L., Nieuwenhuis, S., \& van Boxtel, G. J. M. (2005). Mediofrontal negativities in the absence of responding. Cognitive Brain Research, 25, 777-787.
EriKsen, B. A., \& ERIKSEN, C. W. (1974). Effects of noise letters upon identification of a target letter in a nonsearch task. Perception \& Psychophysics, 16, 143-149.

Falkenstein, M., Hohnsbein, J., Hoormann, J., \& Blanke, L. (1990). Effects of errors in choice reaction tasks on the ERP under focused and divided attention. In C. H. M. Brunia, A. W. K. Gaillard, \& A. Kok (Eds.), Psychophysiological brain research (pp. 192-195). Tilburg: Tilburg University Press.

Falkenstein, M., Hoormann, J., Christ, S., \& Hohnsbein, J. (2000). ERP components on reaction errors and their functional significance: A tutorial. Biological Psychology, 51, 87-107.

Fiehler, K., Ullsperger, M., \& von Cramon, D. Y. (2005). Electrophysiological correlates of error correction. Psychophysiology, 42, 72-82.

Gehring, W. J., Goss, B., Coles, M. G. H., Meyer, D. E., \& Donchin, E. (1993). A neural system for error detection and compensation. Psychological Science, 4, 385-390.

Gehring, W. J., \& Willoughby, A. R. (2004). Are all medial frontal negativities created equal? Toward a richer empirical basis for theories of action monitoring. In M. Ullsperger \& M. Falkenstein (Eds.), Errors, conflicts, and the brain: Current opinions on performance monitoring (pp. 14-21). Leipzig: MPI of Human Cognitive and Brain Sciences.

Gratton, G., Coles, M. G. H., \& Donchin, E. (1983). A new method for off-line removal of ocular artifact. Electroencephalography \& Clinical Neurophysiology, 55, 468-484.

Holroyd, C. B., \& Coles, M. G. H. (2002). The neural basis of human error processing: Reinforcement learning, dopamine, and the errorrelated negativity. Psychological Review, 109, 679-709.

Krigolson, O. E., \& Holroyd, C. B. (2006). Evidence for hierarchical error processing in the human brain. Neuroscience, 137, 13-17.

LuU, P., Flaisch, T., \& TuCKer, D. M. (2000). Medial frontal cortex in action monitoring. Journal of Neuroscience, 20, 464-469.

LUU, P., \& TUCKER, D. M. (2001). Regulating action: Alternating activation of midline frontal and motor cortical networks. Clinical Neurophysiology, 112, 1295-1306.

Luu, P., Tucker, D. M., \& MakeIG, S. (2004). Frontal midline theta and the error-related negativity: Neurophysiological mechanisms of action regulation. Clinical Neurophysiology, 115, 1821-1835.

Manthey, S., Schubotz, R. I., \& von Cramon, D. Y. (2003). Premotor cortex in observing erroneous action: An fMRI study. Cognitive Brain Research, 15, 296-307. 
Mecklinger, A., \& UllsPerger, P. (1995). The P300 to novel and target events: A spatiotemporal dipole model analysis. NeuroReport, $\mathbf{7}$, 241-245.

Miltner, W. H. R., Brauer, J., Hecht, H., Trippe, R., \& Coles, M. G. H. (2004). Parallel brain activity for self-generated and observed errors. In M. Ullsperger \& M. Falkenstein (Eds.), Errors, conflicts, and the brain: Current opinions on performance monitoring (pp. 124-129). Leipzig: MPI of Human Cognitive and Brain Sciences.

Ridderinkhof, K. R., Ullsperger, M., Crone, E. A., \& NieuwenHUIS, S. (2004). The role of the medial frontal cortex in cognitive control. Science, 306, 443-447.

Ullsperger, M., Volz, K. G., \& von Cramon, D. Y. (2004). A common neural system signaling the need for behavioral changes. Trends in Cognitive Sciences, 8, 445-446.

Ullsperger, P., \& Mecklinger, A. (1996). P300 as index of cognitive adaptation. In C. Ogura, Y. Koga, \& M. Shimokochi (Eds.), Recent advances in event-related brain potential research (pp. 47-51). Amsterdam: Elsevier.

van Schie, H. T., Mars, R. B., Coles, M. G. H., \& Bekkering, H. (2004). Modulation of activity in medial frontal and motor cortices during error observation. Nature Neuroscience, 7, 549-554.

Yeung, N., Bogacz, R., Holroyd, C. B., Nieuwenhuis, S., \& Cohen, J. D. (2007). Theta phase resetting and the error-related negativity. Psychophysiology, 44, 39-49.

Yordanova, J., Falkenstein, M., Hohnsbein, J., \& Kolev, V. (2004). Parallel systems of error processing in the brain. NeuroImage, 22, 590-602.

(Manuscript received December 22, 2006; revision accepted for publication August 23, 2007.) 